\title{
PERFIL SENSORIAL DE EMPANADO DE PESCADO
}

Marlice Salete Bonacina*

Rosa de Oliveira Treptow*

Maria Isabel Queiroz ${ }^{* * *}$

\begin{abstract}
O estudo teve por objetivo determinar o perfil sensorial de empanado de pescado, assim como avaliar as alterações das propriedades sensoriais deste produto, em função do tempo de armazenamento. Para obtenção do produto, o músculo da corvina (Micropogonias furnieri) foi triturado e lavado por 30 segundos em água corrente. Após, a polpa foi colocada em cutter e adicionada dos demais ingredientes para homogeneização. A massa foi moldada em formas de alumínio $4 \times 4 \mathrm{~cm}$ e congelada a $-18^{\circ} \mathrm{C}$, para ser submetida a etapa de empanamento e pré-fritura por 30 segundos sob temperatura de $180^{\circ} \mathrm{C}$. O empanado recém elaborado e aquele armazenado por 120 dias a $-18^{\circ} \mathrm{C}$ foram avaliados segundo a Análise Descritiva Quantitativa a partir de oito julgadores treinados. As amostras foram fritas em óleo de soja por três minutos e, então, servidas de forma monádica com três repetições. A análise estatística dos dados foi realizada utilizando o Software Estatística 6.0. Dentre os atributos avaliados, a aparência, o sabor e a textura foram de maior relevância na caracterização do empanado. Em relação ao tempo de armazenamento, verificou-se que apenas o termo granulometria sofreu influência significativa. O empanado foi caracterizado pela cor amarela descrita como bege, gosto salgado, odor, sabor, sabor residual e aroma a pescado e gordura.
\end{abstract}

PALAVRAS CHAVE: PESCADO; VIDA ÚTIL; ANÁLISE DESCRITIVA QUANTITATIVA.

\footnotetext{
* Escola de Química e Alimentos, Universidade Federal do Rio Grande - FURG (e-mail:sbonacina@yahoo. com.br)

** Universidade Federal de Pelotas - UFPeL

***Escola de Química e Alimentos, Universidade Federal do Rio Grande - FURG
} 


\section{INTRODUÇÃO}

Os pescados são importantes constituintes da dieta humana em vários países, uma vez que apresentam uma fonte de proteína de alta qualidade, as quais são comparáveis ao ovo, à carne e ao leite (GONÇALVES e PASSOS, 2003). Porém, no Brasil, seu consumo ainda é baixo, mesmo tendo aumentado nos últimos anos para 11,17 kg por habitante por ano (BRASIL, 2013), valor este bem abaixo do consumo médio mundial expresso como 17,2kg/hab/ano (FAO, 2010).

O baixo consumo de pescado pelos brasileiros é explicado não somente pela falta de hábito, mas por fatores externos que também corroboram para tal efeito, como problemas de logística (distribuição e comercialização), pouca variedade de pescado e produtos de fácil preparo, além do fator socioeconômico (TRONDSEN, 2003).

A elaboração de produtos, utilizando o pescado como matéria prima, é uma alternativa para favorecer o consumo desta fonte proteica, proporcionando uma diversificação para a indústria pesqueira e beneficiando ainda o consumidor com a praticidade no momento do preparo (PASTOUSMADUREIRA et al., 2009; GARCIA-TORCHELSEN et al., 2011; CORTEZ 2010).

Esta constatação é corroborada pelos resultados obtidos por Mitterer-Dalto'e et al., (2012) que avaliaram o potencial de consumo de empanados de pescado mediante determinantes individuais, sugerindo a substituição de pescado fresco por produtos processados, como fator de aumento de consumo. Neste trabalho é indicado ainda, forte potencial de consumo de empanados de pescado por adolescentes associado à necessidade de educação alimentar. Esta classe de produtos acompanha a tendência do mercado e favorece o aproveitamento de espécies como a corvina (Micropogonias furnieri), a qual apresenta baixo valor comercial em relação a outras espécies regionais.

A aceitação de produtos empanados tem sido crescente por parte dos consumidores, uma vez que apresentam aparência, odor e sabor muito apreciados. As características sensoriais de produtos empanados estão relacionadas com a qualidade da matéria-prima utilizada, tempo e temperatura de pré-fritura, bem como o tipo de cobertura (DILL, 2009).

De acordo com Stone \& Sidel (1998) o método que proporciona completa descrição de todas as propriedades sensoriais relevantes é a Análise Descritiva Quantitativa (ADQ). Esta técnica permite discussões no desenvolvimento de termos descritivos, favorecendo a caracterização dos atributos sensoriais perceptíveis do produto em termos quantitativos.

Considerando as informações existente na literatura no que se refere a caracterização sensorial de empanado de pescado, assim como as alterações das propriedades sensoriais que estes sofrem no decorrer do armazenamento, o trabalho se propôs a selecionar e treinar julgadores visando definir o perfil sensorial do empanado de pescado, bem como avaliar a influência do tempo de armazenamento nas características sensoriais deste produto.

\section{MATERIAL E MÉTODOS}

Para elaboração do empanado a corvina (Micropogonias furnieri) foi lavada em água, fileteada e o músculo triturado em moedor de carne manual utilizando disco de $6 \mathrm{~mm}$. A polpa foi submetida ao processo de lavagem em água corrente por 15 segundos, utilizando-se peneira com abertura de $1,25 \mathrm{~mm}$ para retirada do excesso da água. Na sequência a carne foi homogeneizada em um cutter adicionando-se ainda gelo, sal, tripolifosfato de sódio, leite em pó, lactato de sódio, proteína texturizada de soja, gordura, antioxidante e condimentos.

A massa homogeneizada foi moldada em forma de alumínio $4 \times 4 \mathrm{~cm}$, para então ser congelada a $-18^{\circ} \mathrm{C}$. Após o congelamento realizou-se o processo de empanamento, dividido em três etapas. Na primeira etapa o produto passou pelo "predust", em seguida, pelo "batter" diluído em água, na proporção de 1:2 e, por fim, no "breading", para então ser pré-frito a aproximadamente $180^{\circ} \mathrm{C}$, por 30 segundos. Os empanados foram então embalados em sacos de polietileno e congelados a $-18^{\circ} \mathrm{C}$ para posterior realização da análise sensorial. 
Os ingredientes foram obtidos mediante doação da indústria Kerry do Brasil Ltda (Três Corações, Minas Gerais). As farinhas de cobertura: predust, batter e o breading (aplicado ao substrato para conferir o aspecto visual de empanado), foi uma gentileza da Griffith Laboratories Worldwide Ltda (Guarulhos, São Paulo).

\subsection{ANÁLISE DESCRITIVA QUANTITATIVA}

\subsubsection{Seleção de Julgadores}

Para compor a equipe de provadores, foi realizada uma pré-seleção de 13 candidatos, os quais já possuíam experiência em participar de testes sensoriais. Para realizar a seleção final dos julgadores foi realizado o teste Triangular (ABNT, 1993), utilizando duas amostras de empanados que diferiam entre si, quanto ao sabor. Os resultados foram estatisticamente avaliados pela análise sequencial de Wald, segundo o método gráfico (SHIROSE, 1984). O sistema de decisão foi realizado mediante o teste de hipóteses: $H_{0}: p 1<p_{0} ; H_{1}: p_{1}>p_{0}$, e utilizando os valores $p_{0}=0,45$ (máxima inabilidade aceitável), $p_{1}=0,70$ (mínima habilidade aceitável), para os riscos $\alpha=0,05$ (aceita candidato sem acuidade) e $\beta=0,05$ (rejeita candidato com acuidade).

\subsubsection{Desenvolvimento da Terminologia}

Dez julgadores selecionados pelo teste Triangular realizaram o desenvolvimento da terminologia, utilizando-se o método de rede Kelly (MOSKOWITZ, 1983). As amostras utilizadas para gerarem a terminologia foram: empanado de pescado recém-elaborado, com 120 dias de armazenamento e empanado comercial, oriundo do mercado local.

Uma vez desenvolvida à terminologia, a equipe em debate aberto propôs os termos que melhor descrevessem os atributos dos empanados, bem como a definição para cada termo considerado. Os materiais de referência e a definição de cada termo descritivo foram colocados à disposição dos provadores em cada sessão sensorial.

\subsubsection{Avaliação das amostras}

A avaliação dos empanados foi realizada no Laboratório de Análise Sensorial e Controle de Qualidade da Universidade Federal do Rio Grande. As amostras foram codificadas com algarismos de três dígitos e servidas logo após a fritura. A intensidade de cada descritor foi avaliada em cada amostra utilizando a escala não estruturada de nove centímetros, com termos de intensidade ancorados em seus extremos, sendo o mínimo à esquerda e o máximo à direita. Foi utilizado o delineamento em blocos completos balanceados com três repetições, usando a técnica de apresentação monádica.

\subsection{ANÁLISE DOS DADOS}

O tratamento estatístico dos dados foi efetuado segundo o Software Estatística 6.0, nos módulos de Análise de Variância (ANOVA), e Componentes Principais (ACP). Na comparação de médias utilizou-se o teste Tukey, ao nível de erro de $5 \%$.

\section{RESULTADOS E DISCUSSÃO}

\subsection{DESENVOLVIMENTO DA TERMINOLOGIA}

A partir da lista obtida através do método de rede Kelly, a equipe de provadores levantou 37 termos sensoriais, escolhidos em consenso para descrever as similaridades e diferenças entre 
as amostras de empanados. Os termos descritos pelos julgadores para os diferentes atributos sensoriais, suas respectivas definições e os julgador que realizaram as descrições podem ser observado no Tabela 1.

\section{TABELA 1: TERMINOLOGIAS, DEFINIÇÕES E NÚMERO DE JULGADORES PELO MÉTODO DE REDE}

\begin{tabular}{|c|c|c|}
\hline ATRIBUTOS & DEFINIÇÕES & PROVADORES \\
\hline \multicolumn{3}{|l|}{ APARÊNCIA } \\
\hline Cor & Amarelo, descrita como bege contrapondo-se a marrom & $\mathrm{A}, \mathrm{B}, \mathrm{C}, \mathrm{E}, \mathrm{F}, \mathrm{G}, \mathrm{H}, \mathrm{I}, \mathrm{J}$ \\
\hline Uniformidade da cor & Ausência de manchas & $B, C, E, G, H, I$ \\
\hline $\begin{array}{l}\text { Uniformidade da } \\
\text { superfície }\end{array}$ & $\begin{array}{l}\text { Uniformidade da cobertura presença ou não de } \\
\text { partículas }\end{array}$ & $C, E, F, H$ \\
\hline Textura visual & Sensação de textura percebida pela visão & $A, B, C, D, F, G, H, I$ \\
\hline \multicolumn{3}{|l|}{ ODOR } \\
\hline Pescado & Odor a pescado & $\mathrm{A}, \mathrm{B}, \mathrm{C}, \mathrm{D}, \mathrm{E}, \mathrm{F}, \mathrm{G}, \mathrm{H}, \mathrm{I}, \mathrm{J}$ \\
\hline Gordura & Odor a material gorduroso & $\mathrm{A}, \mathrm{B}, \mathrm{C}, \mathrm{D}, \mathrm{E}, \mathrm{F}, \mathrm{G}, \mathrm{H}, \mathrm{I}, \mathrm{J}$ \\
\hline Galinha & Odor que lembra caldo de galinha & $A, D, E, F, G, H, J$ \\
\hline Alho & $\begin{array}{c}\text { Sensação de pungência percebida pela mucosa nasal, } \\
\text { com odor característico a alho }\end{array}$ & $A, E, F, H, I$ \\
\hline Cebola & $\begin{array}{c}\text { Sensação de pungência percebida pela mucosa nasal, } \\
\text { com odor característico a cebola }\end{array}$ & $B, C, F, H$ \\
\hline Vinagre & $\begin{array}{c}\text { Sensação penetrante na mucosa nasal, com odor } \\
\text { característico a cebola }\end{array}$ & G, J, D \\
\hline Camarão & Associado a odor característico a camarão & $\mathrm{H}, \mathrm{D}$ \\
\hline Pipoca & Odor associado a milho torrado, pipoca & $\mathrm{J}$ \\
\hline Queijo & Refere-se a odor característico de queijo & $\mathrm{F}$ \\
\hline \multicolumn{3}{|l|}{ GOSTO } \\
\hline Salgado & Sensação percebida associada a cloreto de sódio & $\mathrm{A}, \mathrm{B}, \mathrm{C}, \mathrm{D}, \mathrm{E}, \mathrm{F}, \mathrm{G}, \mathrm{H}, \mathrm{I}, \mathrm{J}$ \\
\hline Doce & Sensação percebida associada a presença de sacarose & D, G, C \\
\hline Amargo & Sensação percebida associada a solução de cafeína & $\mathrm{F}, \mathrm{I}, \mathrm{E}$ \\
\hline Ácido & Sensação associada a presença de ácidos & $\mathrm{J}, \mathrm{C}$ \\
\hline \multicolumn{3}{|l|}{ AROMA } \\
\hline Pescado & Aroma a pescado & $A, B, C, D, E, F, G, H, I, J$ \\
\hline Gordura & Aroma a material gorduroso & $A, C, I, E, D$ \\
\hline Galinha & Aroma que lembra galinha, canja, caldo de galinha & $\mathrm{D}, \mathrm{H}, \mathrm{E}$ \\
\hline Alho & $\begin{array}{c}\text { Sensação retronasal de pungência, com aroma } \\
\text { característico a alho }\end{array}$ & $B, G, A$ \\
\hline Cebola & $\begin{array}{l}\text { Sensação retronasal de pungência, com aroma } \\
\text { característico a cebola }\end{array}$ & $\mathrm{F}, \mathrm{I}, \mathrm{J}$ \\
\hline Vinagre & $\begin{array}{c}\text { Sensação penetrante retronasal, com aroma } \\
\text { característico a cebola }\end{array}$ & $\mathrm{C}, \mathrm{F}$ \\
\hline Camarão & Sensação associada a aroma característico a camarão & $\mathrm{D}, \mathrm{F}$ \\
\hline Pipoca & Sensação associado ao aroma de milho torrado, pipoca & $\mathrm{J}, \mathrm{F}$ \\
\hline Queijo & Sensação associada ao aroma característico de queijo & $\mathrm{D}$ \\
\hline \multicolumn{3}{|l|}{ SABOR } \\
\hline Pescado & Característico a pescado & $A, B, C, D, E, F, G, H, I, J$ \\
\hline Farinha & Característico a farinha & $E, F, G, J$ \\
\hline Tempero & Sensação que agride a mucosa nasal & $\mathrm{G}, \mathrm{B}, \mathrm{F}, \mathrm{H}, \mathrm{I}$ \\
\hline Queijo & Característico a queijo & $\mathrm{D}, \mathrm{F}$ \\
\hline \multicolumn{3}{|l|}{ TEXTURA } \\
\hline Crocante & $\begin{array}{c}\text { Sensação percebida pela audição associada a força } \\
\text { necessária para quebrar as amostras }\end{array}$ & $B, D, E, F, G, H, I$ \\
\hline Maciez & $\begin{array}{l}\text { Força necessária para deformar as amostras c/ } \\
\text { molares }\end{array}$ & $A, C, J$ \\
\hline Suculência & Quantidade de líquido liberada pela amostra & $A, E, F, G$ \\
\hline \multicolumn{3}{|l|}{ SABOR RESIDUAL } \\
\hline Pescado & $\begin{array}{c}\text { Residual de pescado que fica na boca após a } \\
\text { deglutição }\end{array}$ & $\mathrm{G}, \mathrm{J}, \mathrm{C}, \mathrm{A}, \mathrm{B}$ \\
\hline Gordura & Sensação de recobrimento na cavidade oral & $\mathrm{G}, \mathrm{C}, \mathrm{I}, \mathrm{E}, \mathrm{D}, \mathrm{H}$ \\
\hline Farinha & Residual de farinha que fica na boca após deglutição & $G, J, E, F$ \\
\hline Amargo & Sensação percebida na cavidade oral após deglutição & $\mathrm{F}$ \\
\hline
\end{tabular}


Foi possível verificar que os termos de maior relevância para o atributo aparência foram cor, com percentual de citação de $90 \%$ em função do número total de julgadores, textura visual ( $80 \%)$ e uniformidade da cor (60\%). De acordo com Blumenthal (1996) a aparência, a uniformidade e a crocância são aspectos conferidos pelo processo de fritura, fato este que melhora a apresentação dos produtos.

No que se refere às terminologias para o atributo aroma, notavelmente salientam-se o termo a pescado e gordura com $100 \%$ das citações, seguido do aroma a galinha $(70 \%)$. O termo pescado foi significativo quanto à citação (100\%), para o atributo sabor, seguindo do termo tempero $(50 \%)$. Observou-se ainda que os quatro gostos básicos foram citados, no entanto com total predominância para o gosto salgado (100\%).

Quanto à textura, a crocância foi o termo mais citado (70\%), terminologia que também se reflete na aparência do produto, com equivalente relevância (80\%), o que define uma das propriedades sensoriais de importância para o empanado. Corroborando a afirmação de Llorca et al (2004), os quais reportaram como textura típica de empanado a crocância. Em relação à sensação residual gerada após a deglutição do produto, os termos representativos para este atributo foram gordura $(60 \%)$ e pescado $(50 \%)$.

\subsection{DESEMPENHO DA EQUIPE}

A análise das Figuras 1, 2 e 3 possibilitam avaliar o desempenho da equipe através de suas configurações e respectivas variâncias em relação ao empanado recém elaborado e ao produto armazenado a $-18^{\circ} \mathrm{C}$ por 120 dias.

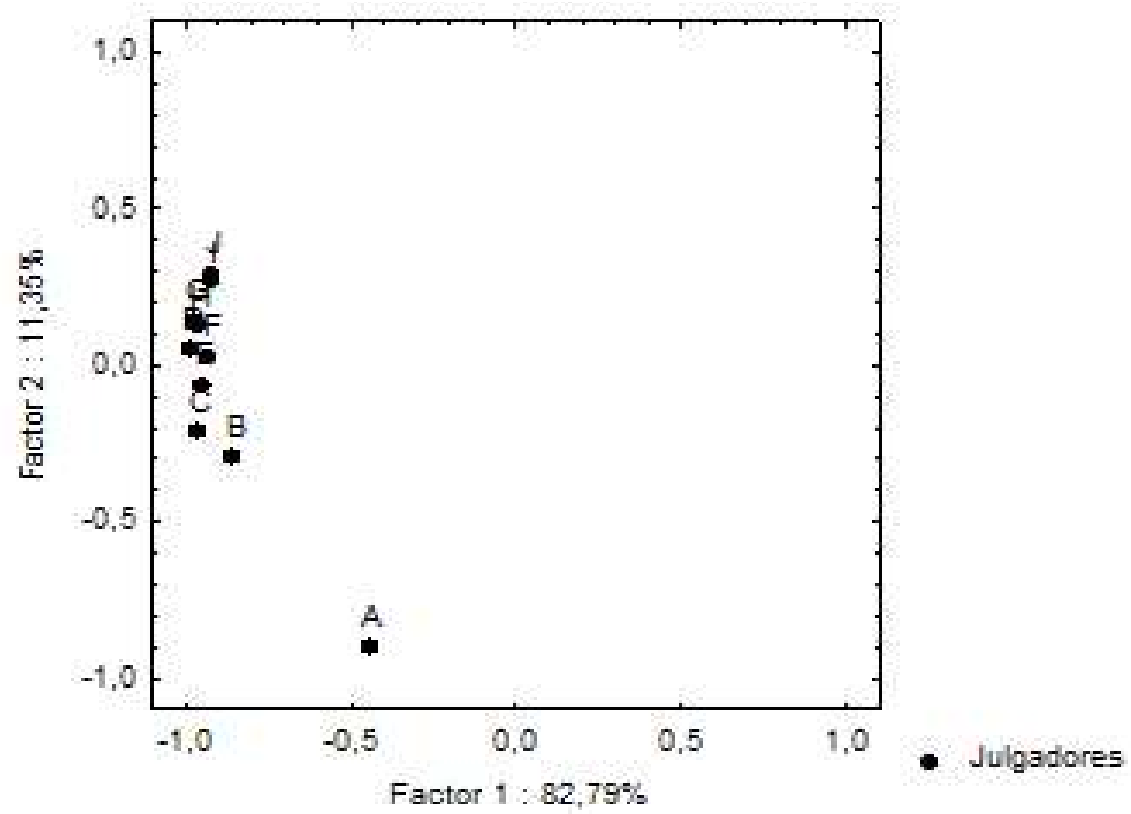

\section{FIGURA 1 - CONFIGURAÇÃO DOS JULGADORES PARA EMPANADO RECÉM- ELABORADO}

Conforme critério estabelecido por Hair (1995), considera-se nesta técnica de análise, suficiente um valor de $60 \%$ para explicação de duas dimensões logo, os percentuais observados nas Figuras $1(93,14 \%)$ e 2 (95,82\%) demonstraram grande representatividade das respostas, enfatizando a validação de sua aplicação. Avaliando-se os resultados registrados verificou-se que o julgador "A" afastou-se dos demais independente da amostra avaliada, diferindo quanto à discriminação dos atributos avaliados em relação à equipe como um todo. Nos empanados armazenados por um período de 120 dias obteve-se comportamento similar ao descrito acima 
para o julgador "B". Portanto, as respostas dos julgadores "A" e "B" não foram consideradas para o tratamento estatístico dos dados, passando a integrar a equipe de avaliação dos empanados, apenas oito dos julgadores iniciais.

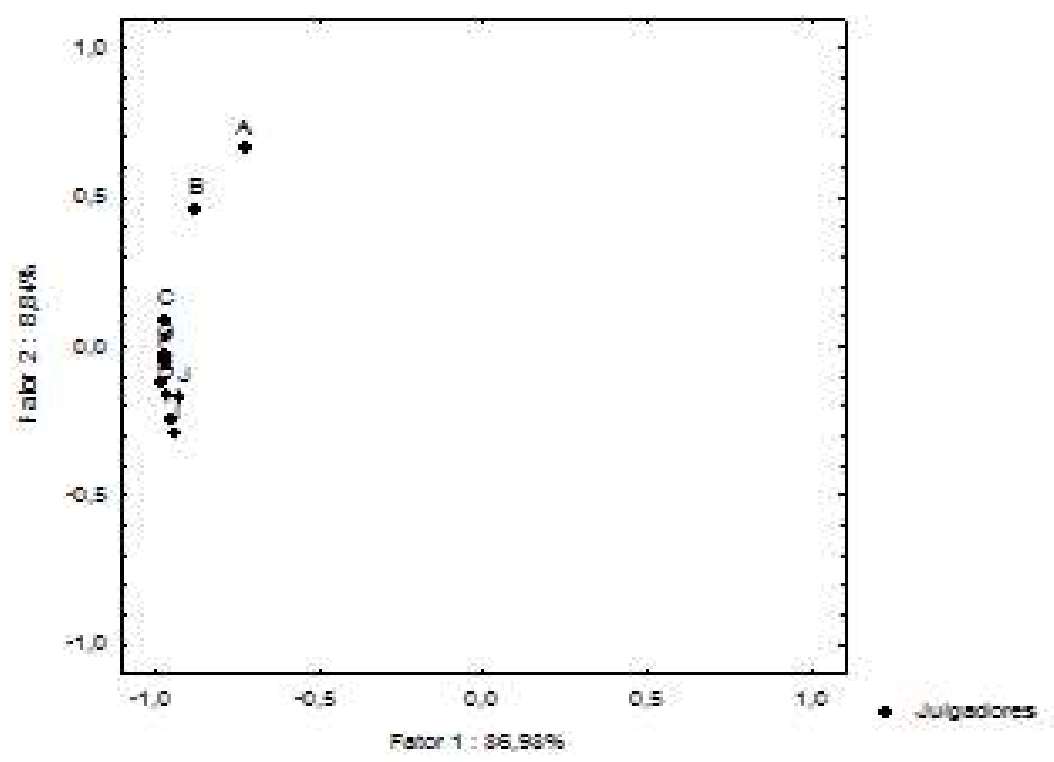

\section{FIGURA 2 - CONFIGURAÇÃO DOS JULGADORES PARA EMPANADO ARMAZENADO POR 120 DIAS}

\subsection{AVALIAÇÃO SENSORIAL DOS EMPANADOS DE PESCADO}

Na Figura 3 observa-se a análise multivariada dos dados sensoriais, realizados mediante Análise de Componentes Principais (ACP) do valor de cada atributo. Na representação gráfica, as configurações dos atributos, terminologias sensoriais dos empanados e a distribuição bidimensional destes foram representadas. Cada eixo explica uma porcentagem da variável total existente entre as amostras.

$\mathrm{Na}$ análise gráfica dos componentes principais, cada amostra está representada geometricamente por um triângulo, onde cada vértice corresponde a uma das triplicatas realizada pela equipe sensorial. Os atributos são representados por vetores, que se encontram voltados para a amostra demonstrando maior ou menor intensidade daquele atributo.

A análise da Figura 4 indica o poder descriminante dos atributos para caracterizar sensorialmente o empanado de pescado. Verifica-se que um percentual de $78,18 \%$ das variações podem ser explicadas pelos dois primeiros fatores (eixos). Isto demonstra que os descritores empregados descriminaram satisfatoriamente as amostras analisadas, considerando os percentuais de variação registrados por outros autores, para diferentes produtos. Morzel et al., (1999) estudando a evolução sensorial de diferentes tipos de salmão fermentado encontraram uma variação de apenas $48,6 \%$ representada pelos dois fatores. Nassu (2010), avaliando a utilização de um protocolo para a análise sensorial de carne bovina, em três diferentes laboratórios, obteve uma variação de $57 \%$ a $68 \%$ de explicação para os dois primeiros fatores.

Cabe salientar, que a primeira dimensão explica $53,25 \%$ das observações, sendo esta associada principalmente aos atributos aparência e sabor. A segunda dimensão explica 24,93\% das variações e é associada principalmente ao odor, sabor residual e textura. Observa-se pela direção dos vetores que a amostra armazenada por 120 dias apresenta maior intensidade, principalmente, dos atributos de gosto, odor e sabor residual, enquanto que a não submetida ao 
processo de armazenamento caracteriza-se pela maior intensidade da aparência, sabor, aroma e textura.

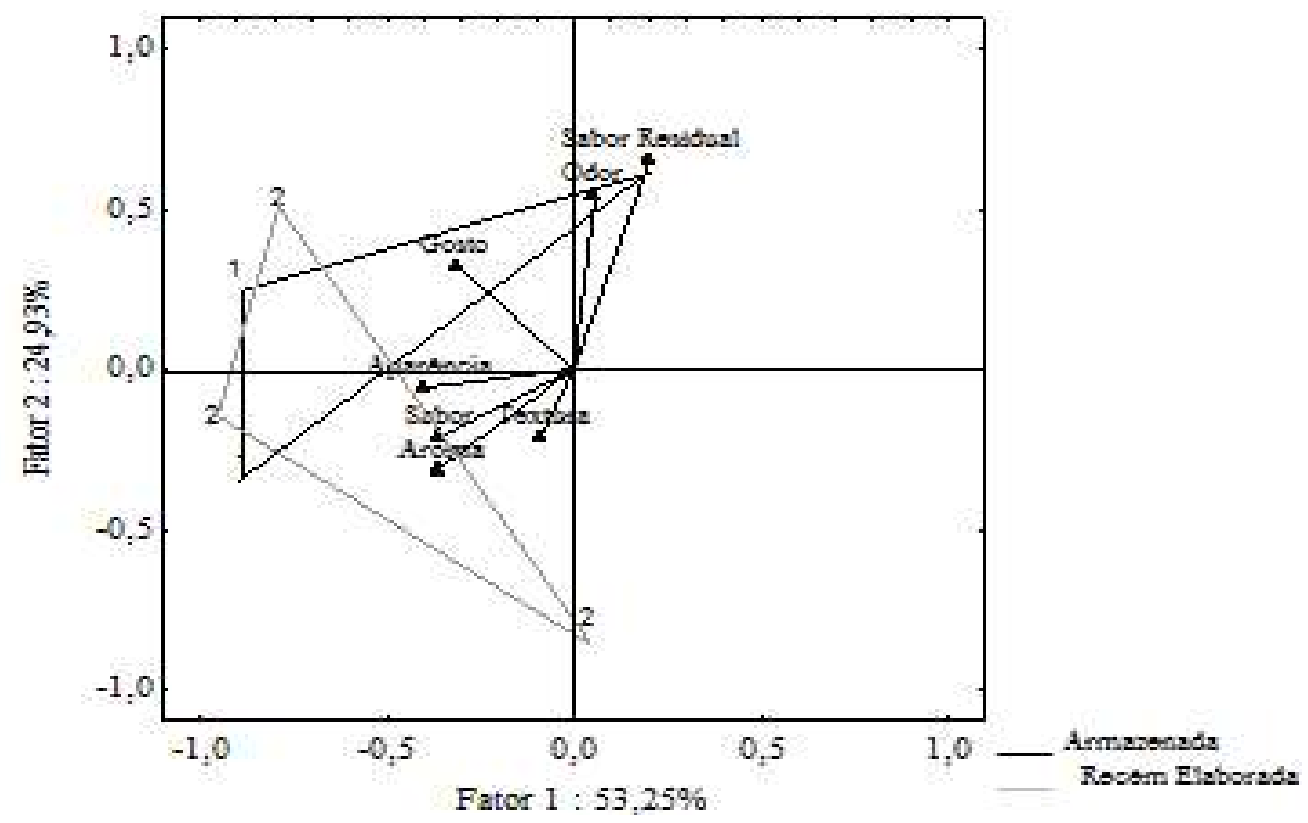

\section{FIGURA 3 - CONFIGURAÇÃO DE DUAS AMOSTRAS DE EMPANADO PARA OITO JULGADORES}

Na Tabela 1 observa-se a representação dos atributos sensoriais avaliados, representados por três dimensões que contribuem com $100 \%$ da variação dos dados. É possível verificar que o fator 1 explica $55,27 \%$ dos dados, para o empanado recém elaborado, com os atributos aroma a cebola, sabor residual amargo, odor a alho e textura crocante como de maior contribuição neste eixo, apresentando valores de $-0,69 ;-0,65 ; 0,65$ e 0,65 respectivamente. Enquanto que para o empanado armazenado por 120 dias o fator 1 explica $58,94 \%$, sendo que os atributos odor a vinagre $(0,89)$, alho $(0,88)$, cebola $(0,77)$ e sabor a farinha $(0,79)$ apresentam maior contribuição (Tabela 2$)$.

TABELA 1 - PORCENTAGEM DE EXPLICAÇÃO DAS VARIÁVEIS DOS DADOS POR FATOR E PRODUTO FORMULADO.

\begin{tabular}{cccc}
\hline Empanado & Fator 1 (\%) & Fator 2 (\%) & Fator 3 (\%) \\
\hline Recém elaborado & 55,27 & 37,97 & 6,76 \\
Armazenado 120 dias & 58,94 & 29,57 & 11,48 \\
\hline
\end{tabular}

O fator 2 explica $37,97 \%$ da variabilidade dos dados para o empanado recém elaborado, conferindo a cor amarelo (bege), odor a camarão e galinha, os atributos de maior contribuição neste eixo, com valores absolutos de $-0,86 ; 0,82$ e 0,75 respectivamente. Em relação ao empanado armazenado, o fator 2 explica $29,57 \%$ da variação e os atributos aroma cebola, galinha e gosto ácido os atributos de maior contribuição -0,77; -0,73 e -0,63 (Tabela 2). Já o fator 3, é responsável por apenas $6,76 \%$ da variabilidade dos dados para o produto recém elaborado e $11,48 \%$ para o produto armazenado por 120 dias. 
TABELA 2 - COORDENADA DAS VARIÁVEIS (CARACTERÍSTICAS SENSORIAIS) NOS EIXOS DOS FATORES 1 A 3 PARA AS DUAS AMOSTRAS DE EMPANADO.

\begin{tabular}{|c|c|c|c|c|c|c|}
\hline \multirow[t]{2}{*}{ Variáveis } & \multicolumn{2}{|c|}{ Recém elaborado } & \multicolumn{2}{|c|}{ Armazenado } & \multicolumn{2}{|l|}{120 dias } \\
\hline & Fator 1 & Fator 2 & Fator 3 & Fator 1 & Fator 2 & Fator 3 \\
\hline \multicolumn{7}{|l|}{ APARÊNCIA } \\
\hline Cor & & $-0,86$ & & 0,26 & & \\
\hline $\begin{array}{l}\text { Uniformidade da } \\
\text { cor }\end{array}$ & & $-0,35$ & & & 0,53 & \\
\hline $\begin{array}{l}\text { Uniformidade da } \\
\text { superfície }\end{array}$ & & $-0,59$ & & & 0,57 & \\
\hline Textura visual & & & $-0,47$ & & 0,46 & \\
\hline Granulometria & & 0,63 & & & & 0,58 \\
\hline \multicolumn{7}{|l|}{ ODOR } \\
\hline Pescado & 0,30 & & & 0,64 & & \\
\hline Gordura & & 0,49 & & & 0,71 & \\
\hline Galinha & & 0,75 & & & & $-0,68$ \\
\hline Alho & 0,65 & & & 0,88 & & \\
\hline Cebola & 0,57 & & & 0,77 & & \\
\hline Vinagre & 0,60 & & & 0,89 & & \\
\hline Camarão & & 0,82 & & & & 0,56 \\
\hline Pipoca & & 0,47 & & & 0,34 & \\
\hline Queijo & & 0,38 & & & & 0,57 \\
\hline \multicolumn{7}{|l|}{ TEXTURA } \\
\hline Crocante & 0,65 & & & & & $-0,63$ \\
\hline Maciez & & & 0,52 & 0,50 & & \\
\hline Suculento & & 0,32 & & 0,65 & & \\
\hline \multicolumn{7}{|l|}{ SABOR } \\
\hline Pescado & & & 0,79 & & $-0,32$ & \\
\hline Farinha & & & $-0,91$ & 0,79 & & \\
\hline Tempero & & $-0,34$ & & & $-0,63$ & \\
\hline Queijo & $-0,55$ & & & & & $-0,64$ \\
\hline \multicolumn{7}{|l|}{ GOSTO } \\
\hline Salgado & 0,62 & & & & $-0,62$ & \\
\hline Doce & & $-0,24$ & & 0,70 & & \\
\hline Amargo & $-0,31$ & & & & & 0,67 \\
\hline Ácido & $-0,52$ & & & & $-0,63$ & \\
\hline \multicolumn{7}{|l|}{$\begin{array}{l}\text { SABOR } \\
\text { RESIDUAL }\end{array}$} \\
\hline Pescado & & $-0,52$ & & & & 0,091 \\
\hline Gordura & & $-0,56$ & & & $-0,35$ & \\
\hline Farinha & 0,60 & & & & $-0,59$ & \\
\hline Amargo & $-0,65$ & & & $-0,55$ & & \\
\hline \multicolumn{7}{|l|}{ AROMA } \\
\hline Pescado & & $-0,67$ & & & & 0,41 \\
\hline Gordura & $-0,40$ & & & & $-0,18$ & \\
\hline Galinha & & $-0,40$ & & & $-0,73$ & \\
\hline Alho & & & $-0,55$ & & $-0,69$ & \\
\hline Cebola & $-0,69$ & & & & $-0,77$ & \\
\hline Vinagre & & 0,15 & & & & \\
\hline Camarão & $-0,61$ & & & & $-0,50$ & \\
\hline Pipoca & & 0,63 & & $-0,43$ & & \\
\hline Queijo & $-0,26$ & & & $-0,50$ & & \\
\hline
\end{tabular}


Na Tabela 3 é possível verificar o valor médio da intensidade dos diferentes atributos sensoriais avaliados nos empanados recém elaborados e armazenados por 120 dias a $-18^{\circ} \mathrm{C}$. Observa-se que as amostras apresentaram características similares em relação ao atributo aparência, exceto no que se refere à granulometria, pois o empanado armazenado apresentou cobertura com granulometria mais fina, diferindo-se $(p \leq 0,05) d a$ amostra sem armazenamento.

Isto pode ser justificado pela perda da cobertura de maior granulometria, pois os empanados foram pré-fritos por 30 segundos a $180^{\circ} \mathrm{C}$ e após armazenados a $-18^{\circ} \mathrm{C}$. Enquanto que os empanados recém elaborados não foram armazenados após a pré-fritura, pois foram avaliados sensorialmente logo após sua elaboração, portanto submetidos à fritura por mais três minutos a $180^{\circ} \mathrm{C}$. Aspecto este que pode ter favorecido a melhor aderência da farinha de cobertura, pois conforme Barbut (2002) a fritura tem como objetivo aderir os componentes do sistema de empanamento e recomenda uma temperatura de $195^{\circ} \mathrm{C}$ por no mínimo um minuto de imersão.

Além disso, os empanados armazenados foram submetidos a uma maior manipulação, aspecto este que pode favorecer o desprendimento da farinha de cobertura do produto (MOURA et al., 2006). Outro fator importante é a umidade da superfície do produto, pois se esta for excessiva, pode trazer um problema na adesão do líquido de empanamento (FISZMAN \& SALVADOR, 2003).

O tempo de armazenamento não influenciou na textura dos empanados, porém cabe destacar que o sistema de cobertura favorece este atributo sensorial, pois a crocância e a maciez foram os termos que mais se destacaram nos produtos avaliados. Este resultado pode ter ocorrido devido ao sistema de cobertura utilizado, pois segundo GL (2002) o tipo de cobertura, principalmente o breading, utilizado no empanamento pode favorecer a crocância do produto, além da retenção desta por um período maior de tempo.

Além disto, o sistema de cobertura age como barreira a perda de umidade, garantindo um produto final macio no interior e ao mesmo tempo crocante (FIZMAN \& SALVADOR, 2003; ALBERT et al, 2009). A crocância também pode ser destacada devido ao produto não ter absorvido quantidade excessiva de gordura, pois a absorção excessiva de gordura durante o cozimento pode comprometer a crocância do empanado SAGUI (2003); WEBER (2008).

Cabe ressaltar, que não existe uma formulação exata para as farinhas de empanamento, estas dependem do substrato do alimento e das características sensoriais desejada do produto. As fórmulas devem ser flexíveis para permitir uma adaptação máxima no desenvolvimento de produtos LOEWE (1990).

Em relação ao odor, gosto, sabor, aroma e sabor residual foi possível verificar comportamento semelhante $(p \geq 0,05)$ entre as amostras de empanado, ou seja, o tempo de armazenamento do produto não influenciou nestes atributos sensoriais.

Este fato pode ser justificado pela lavagem realizada na polpa do pescado antes da elaboração dos empanados, pois, a lavagem além de clarear a polpa, tem o objetivo secundário de remover componentes naturais da carne do pescado, que podem acelerar a deterioração (oxidação lipídica e microrganismos) durante a estocagem a baixas temperaturas, como proteínas solúveis em água, sangue e outros componentes (GONÇALVEZ e PASSOS, 2003; CHAIJAN et al., 2004; LIN et al., 2005; PHATCHARAT et al., 2006; BALANGUE e BENJAKUL, 2009). 
TABELA 3 - MÉDIA DAS INTENSIDADES DOS TERMOS DESCRITOS

PARA OS EMPANADOS

\begin{tabular}{|c|c|c|c|}
\hline Atributos & & $\begin{array}{c}\text { Recém } \\
\text { elaborado }\end{array}$ & $\begin{array}{c}\text { Armazenada por } 120 \\
\text { dias }\end{array}$ \\
\hline \multirow{5}{*}{ APARÊNCIA } & Cor beje & $7,37^{a}$ & $7,24^{a}$ \\
\hline & Uniformidade da cor & $6,27^{a}$ & $5,88^{a}$ \\
\hline & $\begin{array}{l}\text { Uniformidade da } \\
\text { superfície }\end{array}$ & $5,63^{a}$ & $5,96^{a}$ \\
\hline & Textura visual & $6,99^{a}$ & $6,77^{a}$ \\
\hline & Ganulometria & $5,15^{\mathrm{a}}$ & $4,01^{b}$ \\
\hline \multirow{9}{*}{ ODOR } & Pescado & $5,22^{a}$ & $4,60^{a}$ \\
\hline & Gordura & $4,88^{a}$ & $4,58^{a}$ \\
\hline & Galinha & $3,40^{a}$ & $3,77^{a}$ \\
\hline & Alho & $3,03^{a}$ & $2,96^{a}$ \\
\hline & Cebola & $3,43^{a}$ & $3,76^{a}$ \\
\hline & Vinagre & $2,14^{a}$ & $2,41^{a}$ \\
\hline & Camarão & $1,98^{a}$ & $1,88^{a}$ \\
\hline & Pipoca & $2,51^{a}$ & $2,01^{a}$ \\
\hline & Queijo & $1,97^{a}$ & $1,84^{a}$ \\
\hline \multirow{3}{*}{ TEXTURA } & Crocância & $6,11^{a}$ & $6,68^{a}$ \\
\hline & Maciez & $6,78^{a}$ & $6,19^{a}$ \\
\hline & Suculência & $5,92^{\mathrm{a}}$ & $5,47^{a}$ \\
\hline \multirow{4}{*}{ GOSTO } & Salgado & $5,36^{a}$ & $5,03^{a}$ \\
\hline & Doce & $1,72^{\mathrm{a}}$ & $1,84^{a}$ \\
\hline & Amargo & $1,17^{a}$ & $1,30^{\mathrm{a}}$ \\
\hline & Ácido & $1,59^{a}$ & $1,44^{a}$ \\
\hline \multirow{4}{*}{ SABOR } & Pescado & $6,41^{a}$ & $5,60^{a}$ \\
\hline & Farinha & $1,84^{a}$ & $2,10^{a}$ \\
\hline & Tempero & $4,38^{a}$ & $3,74^{a}$ \\
\hline & Queijo & $2,11^{\mathrm{a}}$ & $1,84^{a}$ \\
\hline \multirow{4}{*}{$\begin{array}{c}\text { SAB. } \\
\text { RESIDUAL }\end{array}$} & Pescado & $5,87^{a}$ & $5,32^{a}$ \\
\hline & Gordura & $5,46^{a}$ & $5,05^{\mathrm{a}}$ \\
\hline & Farinha & $1,82^{a}$ & $2,27^{a}$ \\
\hline & Amargo & $1,23^{a}$ & $1,25^{\mathrm{a}}$ \\
\hline \multirow{9}{*}{ AROMA } & Pescado & $5,82^{a}$ & $5,59^{a}$ \\
\hline & Gordura & $4,99^{a}$ & $5,02^{a}$ \\
\hline & Galinha & $3,44^{a}$ & $3,26^{a}$ \\
\hline & Alho & $2,64^{a}$ & $2,70^{a}$ \\
\hline & Cebola & $2,56^{a}$ & $2,50^{a}$ \\
\hline & Vinagre & $1,46^{a}$ & $1,47^{a}$ \\
\hline & Camarão & $2,30^{\mathrm{a}}$ & $2,01^{a}$ \\
\hline & Pipoca & $1,99^{a}$ & $2,12^{\mathrm{a}}$ \\
\hline & Queijo & $1,98^{a}$ & $1,83^{a}$ \\
\hline
\end{tabular}

Letra iguais na mesma coluna não apresentam diferença ( $p £ 0,05)$ 
Os empanados apresentaram maior intensidade, principalmente, para os termos de pescado e gordura. Segundo Selli e Cayhan, 2009), o peixe tem um aroma característico, o qual é influenciado pela espécie e por condições de manipulação e armazenamento após a captura. Ainda conforme estes autores, importantes compostos de aroma, característicos de pescado, são gerados principalmente por reações de oxidação enzimática e auto-oxidação de lipídeos.

Serfert et al. (2010) identificaram o composto 1-penteno-3-ol como um dos voláteis responsáveis pelo odor de óleo de peixe caracterizado sensorialmente por termos como pescado.

A intensidade da gordura se justifica, principalmente, pelo fato do empanado ser frito, assim, a gordura se torna um ingrediente importante, pois o óleo é absorvido no processo de cozimento do alimento após a perda parcial de água por evaporação (SAGUY e DANA, 2003; WEBER et al., 2008).

Outro termo que merece destaque nos atributos sensoriais é o tempero, o qual pode ter sido oriundo da adição, na formulação do produto, de pimenta branca, alho e cebola em pó. $O$ tempero também pode ter sido quantificado devido à presença do mesmo nas farinhas de cobertura, pois segundo BORTOLUZZI (2006) o sistema de cobertura pode ser um carreador de condimentos.

\section{CONCLUSÔES}

O tempo de armazenamento estudado, não influenciou significativamente nas características sensoriais do empanado de pescado, produto este que pode ser caracterizado sensorialmente por apresentar cor amarela descrita como bege pelos julgadores, gosto salgado, odor, sabor, sabor residual e aroma a pescado e gordura.

\section{ABSTRACT}

\section{SENSORY PROFILE OF BREADED FISH}

The study aimed to determine the sensory profile of breaded fish, as well as to assess the change of sensory properties of this product, due to storage time. To obtain the product, the muscle of Whitemouth croaker (Micropogonias furnieri) was triturated and washed for 30 seconds in running water. After that, the pulp was placed in cutter and added the other ingredients for homogenization. The mass was placed then in aluminum molds $4 \times 4 \mathrm{~cm}$ and frozen at $-18^{\circ} \mathrm{C}$ to be submitted to the breaded and pre-frying stage for 30 seconds at a temperature of $180^{\circ} \mathrm{C}$. The breaded recently elaborated and the one stored for 120 days at $-18^{\circ} \mathrm{C}$ were assessed according to the Quantitative Descriptive Analysis by eight trained judges. The samples were fried in soy oil for three minutes and, then, served in monadic form with three repetitions. The statistical analysis of the data was made using the Software Statistics 6.0. Among the attributes assessed, the appearance, flavor and texture had grater relevance in the characterization of the breaded. Regarding the time of storage, we verified that only the term granulometry had significant influence. The breaded was characterized by yellow color described as beige, salt taste, fish and fat scent, flavor, aroma and residual flavor.

KEY WORDS: FISH, SHELF LIFE, QUANTITATIVE DESCRIPTIVE ANALYSIS.

\section{REFERENCIAS}

1 ALBERT, A.; PEREZ-MUNUERA, I.; QUILES, A.; SALVADOR, A.; FISZMAN, S. M.; HERNANDO, I. Adhesion in fried battered nuggets: Performance of different hydrocolloids as predusts using three cooking procedures. Food Hydrocolloids, v. 23, p.1443-1448, 2009. 
2 ASSOCIAÇÃO BRASILEIRA DE NORMAS TÉCNICAS. NBR 12995: teste triangular em análise sensorial dos alimentos e bebidas. São Paulo, 1993. 5p.

3 BALANGE, A. K.; BENJAKUL, S. Effect of oxidised tannic acid on the gel properties of mackerel (Rastrelliger kanagurta) mince and surimi prepared by different washing processes. Food Hydrocolloids, v.23, p.1693-1701, 2009.

4 BARBUT, S. Poultry Products Processing: An Industry Guide. Boca Raton: CRC Press LLC, 2002. 548p.

5 BLUMENTHAL, M. M. Frying technology. In: BAILEY, A. E. Bailey's industrial oil \& fat products. New York: John Wiley, v. 3, 1996. p. $429-481$.

6 BORTOLUZZI, R. C. Empanados. In: R. OLIVO (ed.), O mundo do frango: cadeia produtiva da carne de frango. Criciúma, Ed. Do Autor, 2006.p. 481-494.

7 BRASIL. Ministério da Pesca e Aquicultura - MPA, Boletim Estatístico da Pesca e Aquicultura 2008-2009, Brasil 2010, $101 \mathrm{p}$.

8

9 CHAIJAN, M.; BENJAKUL, S.; VISESSANGUAN, W.; FAUSTMAN, C. Characteristics and gel properties of muscles from sardine (Sardinella gibbosa) and mackerel (Rastrelliger kanagurta) caught in Thailand. Food Research International, v.37, p.1021-1030, 2004.

10 CORTEZ NETTO, J.P; BOSCOLO, W.R.; FEIDEN, A.; MALUF, M. L. F.; FREITAS, J.M.A.; SIMÕES, M.R. Formulação, analises microbiológicas, composição centesimal e aceitabilidade de empanados de jundiá (Rhamdia quelen), pacu (Piaractus mesopotamicus) e tilápia (Oreochromis niloticus. Rev. Inst. Adolfo Lutz, v.69, n.2, p. 181-187, 2010.

11 DILL, D.D.; SILVA, A.P.; LUVIELMO, M.M. Processamento de empanados: sistemas de cobertura. Estudos Tecnológicos - v. 5, n.1, p.33-49, 2009.

12 FISZMAN, S. M.; SALVADOR, A. Recent developments in coating batters. Trends in Food Science \& Technology, v.14, p.399-402, 2003

13 FOOD AND AGRICULTURE ORGANIZATION OF THE UNITED NATION (FAO). Statistical databases. Disponível em: <http://www.fao.org>. Acesso em: novembro de 2013.

14 GARCIA-TORCHELSEN, L. Utilização de base protéica de anchoita (Engraulis anchoita) na elaboração de empanado, 120-140. Em: Caracterização do Estoque de Anchoita (Engraulis anchoita) da Região Sul do Brasil e Utilização desta Matéria-prima na Elaboração de Produto de Alto Valor Agregado.2011.181f. Tese de Doutorado, Escola de Química e Alimentos, Universidade Federal do Rio Grande Rio Grande, 2011.

15 GL-LABORATORIES WORLDWIDE. Guia completo para sistemas de cobertura. Guarulhos, Ed. do Autor, 2002. 41 p.

16 GONÇALVES, A. A.; PASSOS, M. G. Uso da enzima transglutaminase na elaboração de um produto reestruturado à base de pescado. Revista Nacional da Carne, São Paulo, v. 28, n.317, p. 123-132. 2003.

17 HAIR, F.; ANDERSON, R.E.; TATHAM, R.L.; BLACKMAN, W.C. Multivariate Data Analysys: whit readings. 4ed. Englewood Cliffs Prentice-Hall, 1995, 757p.

18 LIN, S. B.; CHEN, L. C.; CHEN, H. H. The change of thermal gelation properties of horse mackerel mince led by protein denaturation occurring in frozen storage and consequential air floatation wash. Food Research International, v.38, p.1927, 2005.

19 LLORCA, E. , HERNANDO, I., PÉREZ-MUNUERA, I., QUILES, A., LARREA, V., FISZMAN,S. M., LLUCH, M. A. Microstructural Sttudy of Fronze Batter-Coated Squid Rins Prepared byan Innovative Process Without a Pre-Fring Step. Food Hydrocolloids v.19, n.2, p.297-302, 2005.

20 LOEWE, R.. Ingredients selection for batters systems. In: K. KULP; R. LOEWE (eds.), Batters and breadings in food processing. St.Paul, American Association of Cereal Chemists, Inc., 1990. p.11-28.

21 MITTERE-DALTOÉ, M.L.; LATORRES, J.M.; CARBONERA, N.; PASTOUS-MADUREIRA, L.S.; QUEIROZ, M.I. Potencial de inserção de empanados de pescado na merenda escolar mediante determinantes individuais. Ciência Rural, v.42, n.11, p.2092-2098, 2012.

22 MORZEL, M., SHEEHAN, E. M., DELAHUNTY, C. M., ARENDT, E. K. (1999) Sensory evaluation of lightly preserved salmon using free-choice profiling International Journal of Food Science and Technology, v. 34, p. 115-123.

23 MOSKOWITZ, H. R. Product testing and sensory evalution of foods. Westport: Food \& Nutrition Press, 1983, 605p.

24 MOURA, C.P.; TIMPONE, L. T.; BARCARO, P.; LIMA, R.C.A. Produtos Reestruturados. Revista Nacional da Carne, p.90-96, 2006

25 NASSU, R.T.; BORBA, R. VERRUMA-BERNARDI, M.G. Validação de protocolo sensorial para avaliação de carne bovina. Braz. J. Food Technol. N.6, p. 152-160, 2010. 
26 PASTOUS MADUREIRA, L. S.; CASTELLO, J. P.; PRENTICE-HERNÁNDEZ, C.; QUEIROZ, M. I.; ESPÍRITO SANTO, M. L.; RUIZ, W. A.; RAGGI ABDALLAH, P.; HANSEN, J.; BERTOLOTTI, M. I.; MANCA, E.; YEANNES, M. I.; AVDALOV, N.; FERNÁNDEZ AMORÍN, S. Current and potential alternative food uses of the Argentine anchoita (Engraulis anchoita) in Argentina, Uruguay and Brazil. In: HASAN, M. R.; HALWART. M. (Eds.). Fish as Feed Inputs for Aquaculture: Practices, Sustainability and Implications. Rome: FAO, 2009. p. 269-287. FAO Fisheries and Aquaculture Technical Paper, n. 518, 2009.

27 PHATCHARAT, S.; BENJAKUL, S.; VISESSANGUAN, W. Effects of washing with oxidizing agents on the gel-forming ability and physicochemical properties of surimi produced from bigeye snapper (Priacanthus tayenus). Food Chemistry, v.98, p.431-439, 2006.

28 SAGUY, I.S.; DANA, D. Integrated approach to deep fat frying: Engineering, nutrition, health and consumer aspects. Journal of Food Engineering, v.56, p.143-152, 2003.

29 SELLI, S.; CAYHAN, G. G. Analysis of volatile compounds of wild gilthead sea bream (Sparus aurata) by simultaneous distillation-extraction (SDE) and GC-MS. Microchemical Journal, v.93, p.232-235, 2009.

30 SERFERT, Y.; DRUSCH, S.; SCHWARZ, K. Sensory odour profiling and lipid oxidation status of fish oil and microencapsulated fish oil. Food Chemistry, v.123, p.968-975, 2010.

31 SHIROSE, I.; MORI, E. E. M. Aplicação da análise seqüencial á seleção de provadores, pelo teste triangular. ITAL, v.14, p. 39-55, 1984.

32 STONE, H., SIDEL, J. L. Quantitative Descriptive Analysis: Developments, Applications, and the Future. Food Techology v. 52, n. 8, p. $48-52,1998$

33 TRONDSEN T, SCHOLDERER J, LUND E, EGGEN AE. Perceived barriers to consumption of fish among Norwegian women. Appetite, v.41, n.3, p.301-314, 2003.

34 WEBER, J.; BOCHI, V. C.; RIBEIRO, C. P.; VICTÓRIO, A. M.; Emanuelli, T. Effect of different cooking methods on the oxidation, proximate and fatty acid composition of silver catfish (Rhamdia quelen) fillets. Food Chemistry, v. 106, p. 140146, 2008. 